\title{
Effect of the ExactCare medication care management model on adherence, health care utilization, and costs
}

Kanaka D Shetty, MD, MS; Annie Y Chen, DDS, MS; Adam J Rose, MD, MSc; and Harry H Liu, PhD

\section{What is already known about this subject}

- Multimorbidity and polypharmacy are common in the United States and are associated with greater risk of disease-related complications and higher health care costs.

- Individual interventions to improve medication management, such as blister packs or pharmacist-led management, have shown promise in several earlier studies but may be insufficient for long-term improvement.

\section{What this study adds}

- One year of ExactCare participation was associated with statistically significant increases in adherence to statins and antihypertensive drugs.

- ExactCare participation was associated with statistically significant decreases in SNF admission rates, inpatient days, and SNF days.

- ExactCare participation was associated with increases in prescription drug costs but reductions in total costs of care of approximately $\$ 2,400$ per member per year.

\section{Author affiliations \\ Kanaka D Shetty, MD, MS, and Annie Y Chen, DDS, MS, RAND Corporation, Santa \\ Monica, CA. Adam J Rose, MD, MSc, Hebrew University School of Public Health, Jerusalem, Israel. Harry H Liu, PhD, RAND Corporation, Boston, MA.}

AUTHOR CORRESPONDENCE: Harry H Liu, 617.338.2059 x8638; hliu@rand.org

J Manag Care Spec Pharm. 2021;27(5):574-85

Copyright $@ 2021$, Academy of Managed Care Pharmacy. All rights reserved.

outcomes included emergency department (ED) visits, hospitalizations, and skilled nursing facility (SNF) admissions, hospitalization days, and SNF days. Cost outcomes included total cost of care, prescription drug cost, hospital inpatient cost, and SNF cost.

RESULTS: ExactCare patients $(\mathrm{N}=701)$ were propensity-matched to comparison patients $(\mathrm{N}=1,395)$ using the nearest $1: 2$ match approach, with an average follow-up period of 6.6 and 5.4 years for ExactCare and comparison patients, respectively. One year of ExactCare participation was associated with statistically significant increases in adherence to statins (8.4 percentage points; $P<0.001)$ and antihypertensive drugs (4.9 percentage points; $P<0.001)$, but the increase in adherence for diabetes drugs was not statistically significant. ExactCare participation was associated with statistically significant decreases in SNF admission rates (-67 SNF 
stays per 1,000 member-years; $P=0.011)$, inpatient days ( -857 days per 1,000 member-years; $P=0.022)$, and SNF days $(-1,801$ days per 1,000 member-years; $P=0.002$ ), but not with the rates of $E D$ visits or hospital admissions. Each year of ExactCare participation was associated with increases in prescription drug costs $(\$ 30$ per-member per month [PMPM]; $P=0.006)$ and decreases in total costs (-\$196 PMPM; $P=0.023)$ and medical costs ( $\$ 226 P M P M ; P=0.008)$, largely attributable to decreases in hospital inpatient costs ( $\$ 119$ PMPM; $P=0.001)$ and SNF costs ( $\$ 30$ PMPM; $P=0.007)$.

CONCLUSIONS: ExactCare's medication care management model was associated with improved medication adherence and an approximately $\$ 2,400$ per member per year reduction in total cost of care, representing a $5 \%$ reduction in average costs.

Multimorbidity and polypharmacy (usage of 5 or more medications) are common in the United States, ${ }^{1}$ with approximately $40 \%$ of older community-dwelling adults meeting the definition for polypharmacy. ${ }^{2}$ Extreme polypharmacy (concomitant use of 10 or more medications) is also increasingly common, with prevalence rates of more than $30 \%$ being reported, and may be associated with greater risk of disease-related complications and higher costs. ${ }^{3-5}$

The optimal approach to improving medication management for patients using complex regimens is largely unknown. Individual interventions may be effective for improving adherence, including blister packs, ${ }^{6}$ electronic bottle caps, ${ }^{7,8}$ phone or text reminders, ${ }^{9-14}$ use of mailorder pharmacies, ${ }^{15-17}$ and pharmacist-delivered enhanced medication therapy management. ${ }^{18-30}$ However, attempts to help such patients are not uniformly successful: several studies of electronic pill bottles with and without both human intervention and bidirectional text messaging did not substantially improve adherence in patients, ${ }^{11,12,31,32}$ and phone or text reminders have limited evidence for longterm effectiveness. ${ }^{14}$ Given that individual interventions may be insufficient for long-term improvement, high-touch approaches that combine several interventions may be a more effective strategy to reduce complications associated with polypharmacy. In addition to increasing adherence, such approaches also aim to simplify complex medication regimens, reduce barriers to access (such as transportation), and reconcile medication regimens from multiple providers. However, to our knowledge, no previous study has examined the effect of combining all of these features on adherence and outcomes in high-risk elder patients. ${ }^{32-35}$

ExactCare, a health care company that aims to address medication-related and chronic care challenges, has designed and has been implementing a high-touch medication care management approach (hereinafter the ExactCare program) for medically complex high-risk patients over the past decade. Its intervention starts with an initial patient home visit to conduct a comprehensive medication review, assess the patient's home situation, and provide patient education. ${ }^{36}$ After a personal pharmacy team verifies and reconciles all medications from different prescribers, the company prepares and sends 1 or more pouches containing all oral medications (hereinafter ExactPacks) to each patient's home, including over-thecounter medications, along with directions to take them at specific times. Nonoral medications, such as insulin and topical medications, are also shipped to the patient in the same monthly shipment as the ExactPack or more frequently as needed. The pharmacy team reviews medications and manages refills monthly for each patient and monitors adherence, either using a smartphone application or web portal to track usage or by directly assessing medication usage. The pharmacy team also issues reminders by phone, text message, or mobile app to facilitate patients to take their medication on time.

The effectiveness of the ExactCare program on clinical outcomes likely requires improved adherence to pharmaceutical therapy for most medication classes. In turn, medication adherence improvement could potentially lead to better control of chronic medical conditions, leading to fewer exacerbations of such conditions, less health care utilization such as emergency department (ED) visits and hospitalizations, and lower overall health care cost, but presumably with higher prescription drug cost due to the more consistent refills.

However, based on previous studies, it is unknown whether a high-touch approach such as the ExactCare program improves medication adherence and reduces health care resource utilization among patients with multimorbidity and polypharmacy. To answer these questions, we leveraged the medical and prescription drug encounter data of Medicare Advantage beneficiaries from one of the largest private insurers in the United States. Although it is infeasible to assess all medication classes, we examined whether the program improved medication adherence rates for oral medications, including those used for 3 common conditions: hyperlipidemia, hypertension, and diabetes. We then analyzed health care utilization (including ED visits, hospitalizations, skilled nursing facility [SNF] admissions) and costs (prescription drug cost, medical cost, and total cost of care). For both types of analyses, we used a difference-in-differences approach to model these outcomes in ExactCare patients before and after enrollment, compared with similar patients who were not enrolled in ExactCare. 


\section{FIGURE 1 Inclusion Flowchart}

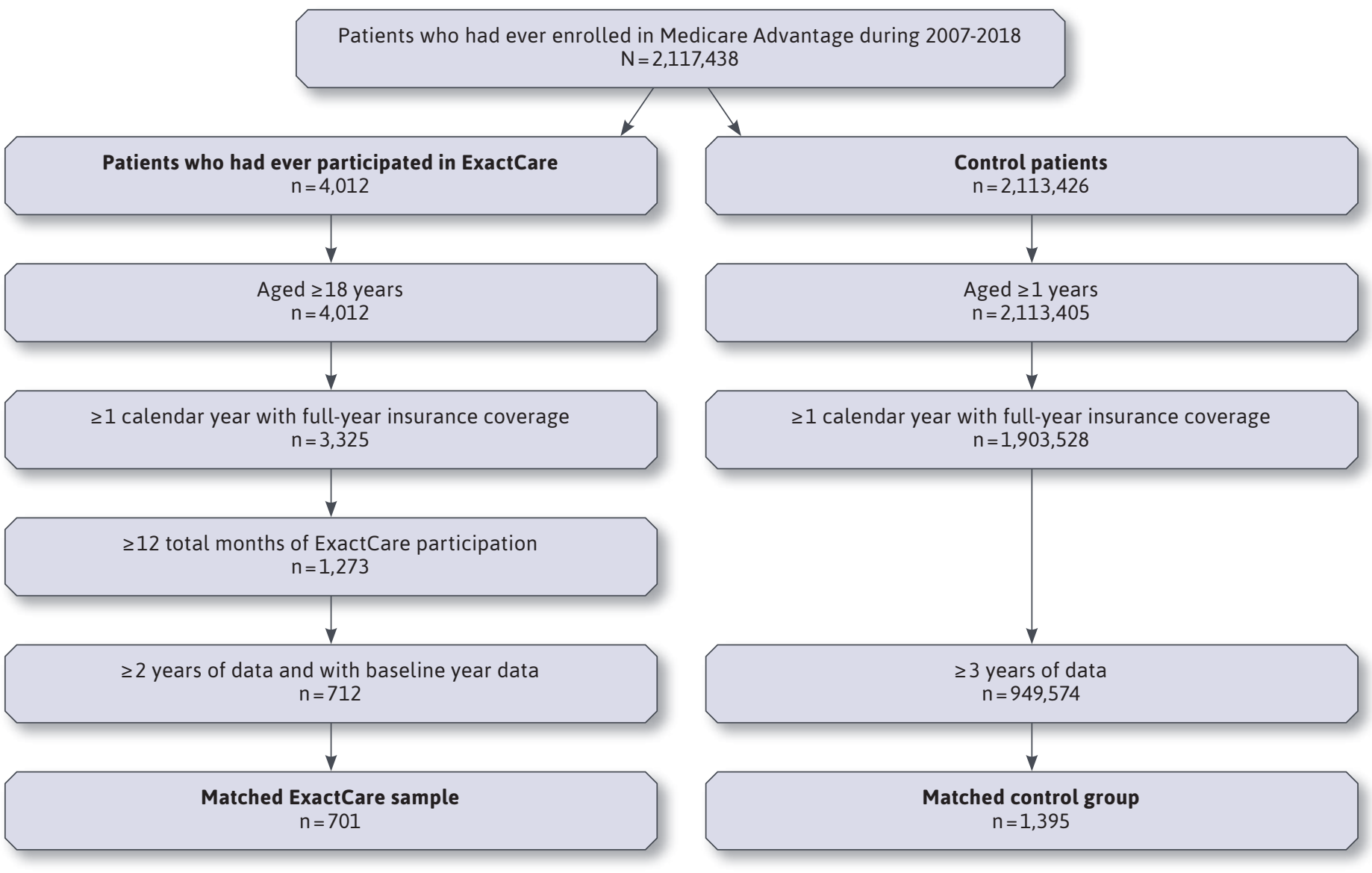

\section{Methods}

\section{DATA SOURCES AND STUDY POPULATION}

Using a national database from a large U.S. insurer, we identified a cohort of Medicare Advantage plan members in 8 states (Illinois, Indiana, Kentucky, Michigan, New Jersey, Ohio, Pennsylvania, Texas) that included both ExactCare participants and nonparticipants from 2007 to 2018. All plan members had the following associated data: demographic information (including age, gender, and race); urban residence; insurance coverage (including Medicaid eligibility and receipt of Medicare Part D low-income subsidies); professional service records (including place of service, diagnosis, and procedure codes); facility service records (including place of service, diagnosis, and procedure codes); and prescription drug event records (including prescription drug name, prescription start and end dates, quantity and strength, Medispan therapeutic class, and pharmacy identifiers).

We identified ExactCare patients using prescription drug event records. Any patient who had at least 1 prescription event record filled in pharmacies solely dedicated to ExactCare patients was considered enrolled into the ExactCare program at some point during the study period, and the rest of the patients were considered comparison patients. Patients were referred to ExactCare by health plans, home health care providers, or primary care providers. Typically, it took about 3 months for a patient to transition into the ExactCare program. During that time, a home assessment, patient education, and medication reconciliation were conducted, and patients may have received some prescriptions from ExactCare. Starting in the fourth month, formal ExactPacks were sent to patients' homes. 


\section{TABLE 1}

\section{Baseline Patient Characteristics Before Propensity Score Matching ${ }^{\mathrm{a}}$}

\begin{tabular}{|c|c|c|c|}
\hline Characteristics & ExactCare & Comparison & P Value \\
\hline \multicolumn{4}{|l|}{ Sociodemographics } \\
\hline Age, mean (SD), years & $72.44 \quad(11.41)$ & $71.81 \quad(9.34)$ & 0.074 \\
\hline Female, $\%$ & 66.99 & 57.44 & $<0.001$ \\
\hline \multicolumn{4}{|l|}{ Race, \% } \\
\hline White & 47.75 & 60.10 & $<0.001$ \\
\hline Black & 41.57 & 10.39 & $<0.001$ \\
\hline Other & 10.67 & 29.51 & $<0.001$ \\
\hline $\begin{array}{l}\text { Dually eligible for Medicare and } \\
\text { Medicaid, \% }\end{array}$ & 25.00 & 7.80 & $<0.001$ \\
\hline $\begin{array}{l}\text { Receipt of Medicare Part D } \\
\text { low-income subsidies, \% }\end{array}$ & 48.17 & 15.60 & $<0.001$ \\
\hline Urban residence, \% & 99.16 & 96.08 & $<0.001$ \\
\hline \multicolumn{4}{|l|}{ Diagnosed medical conditions } \\
\hline HCC score, mean (SD) & $2.28 \quad(1.63)$ & $(1.02)$ & $<0.001$ \\
\hline Diabetes, \% & 24.02 & 15.98 & $<0.001$ \\
\hline Cardiovascular disease, $\%$ & 53.23 & 27.55 & $<0.001$ \\
\hline Stroke, \% & 12.64 & 3.62 & $<0.001$ \\
\hline Chronic lung disorders ${ }^{\mathrm{b}}, \%$ & 33.29 & 13.93 & $<0.001$ \\
\hline Chronic liver disease, $\%$ & 1.69 & 0.86 & 0.017 \\
\hline Renal failure ${ }^{c}, \%$ & 5.06 & 1.70 & $<0.001$ \\
\hline Cancer, \% & 8.01 & 6.36 & 0.072 \\
\hline Dementia, \% & 13.90 & 5.44 & $<0.001$ \\
\hline Major mental health conditions ${ }^{d}, \%$ & 16.15 & 6.74 & $<0.001$ \\
\hline Paralysise,$\%$ & 4.92 & 1.42 & $<0.001$ \\
\hline Transplant, \% & 0.28 & 0.14 & 0.331 \\
\hline HIV, \% & 0.84 & 0.22 & $<0.001$ \\
\hline Autoimmune diseases ${ }^{f}, \%$ & 6.60 & 6.51 & 0.919 \\
\hline
\end{tabular}

\section{INTERVENTION AND COMPARISON GROUPS}

The process for selecting potential intervention and comparison groups is illustrated in Figure 1. For each calendar year, all patients were required to have both 12-month medical and prescription drug insurance coverage and be aged 18 years or older.

For the main analysis, we defined the intervention group as all patients within the study population who during 2007-2018. In addition, all ExactCare patients had to have at least 1 year of data before the first program participation year, which allowed us to conduct a differencein-differences analysis. Patients are followed throughout 2007-2018 and leave only if they died or switched insurers.

We derived the comparison group using similar inclusion criteria: aged 18 years or older and with at least 1 calendar year with full-year medical and prescription drug insurance coverage. In addition, we required patients to have at least 3 years of data, to ensure adequate data availability for propensity matching and for characterizing utilization and cost during the study period. After these exclusions, we identified a cohort of patients that had similar levels of needs to ExactCare patients, using propensity score matching (see details in Analytic Approach).

\section{OUTCOMES}

Medication Adherence. As previously explained, we expected the most proximal effect of ExactCare to be seen in improved adherence to medications. To measure this, we computed adherence rates for ExactCare patients and comparison patients for 3 key medication classes that are subject to validated quality measures and used by the Centers for Medicare and Medicaid Services: antihypertensives (primarily angiotensin-converting enzyme inhibitors and angiotensin receptor blockers), hydroxymethylglutaryl-coenzyme A reductase inhibitors (i.e., statins), and oral diabetic medications (including 8 drug classes) ${ }^{37-39}$ Using the measure specifications defined by Pharmacy Quality Alliance, we computed the proportion of days covered (PDC) for each class (Supplementary Table 1, available in online article), which is the proportion of days that a patient 


\section{TABLE 1 Baseline Patient Characteristics Before Propensity Score Matchinga (continued)}

\begin{tabular}{|c|c|c|c|}
\hline Characteristics & ExactCare & Comparison & P Value \\
\hline \multicolumn{4}{|l|}{ Health care utilization and costs } \\
\hline $\begin{array}{l}\text { Emergency room visits/ } \\
1,000 \text { patient-years, mean (SD) }\end{array}$ & $1,969(4,183)$ & $544(1,721)$ & $<0.001$ \\
\hline $\begin{array}{l}\text { Inpatient admissions/ } \\
1,000 \text { patient-years, mean (SD) }\end{array}$ & $730(1,575)$ & $178 \quad(586)$ & $<0.001$ \\
\hline $\begin{array}{l}\text { Skilled nursing facility stays/ } \\
1,000 \text { patient-years, mean (SD) }\end{array}$ & $256 \quad(717)$ & $41 \quad(287)$ & $<0.001$ \\
\hline Medication classes, mean (SD), $\mathrm{n}$ & $12.33 \quad(6.31)$ & $6.95 \quad(6.11)$ & $<0.001$ \\
\hline PMPM medical cost, mean (SD), USD & $3,097 \quad(6,448)$ & $1,137 \quad(3,125)$ & $<0.001$ \\
\hline $\begin{array}{l}\text { PMPM prescription cost, } \\
\text { mean (SD), USD }\end{array}$ & $513(1,187)$ & $(847)$ & $<0.001$ \\
\hline PMPM total cost, mean (SD), USD & $3,610(6,644)$ & $1,399 \quad(3,317)$ & $<0.001$ \\
\hline \multicolumn{4}{|l|}{ Medication adherence, PDC } \\
\hline Cholesterol medications, \% (SD) & $82.77(18.47)$ & $84.81(18.38)$ & 0.020 \\
\hline Hypertension medications, \% (SD) & $82.93(19.28)$ & $86.72(17.36)$ & $<0.001$ \\
\hline Diabetes medications, \% (SD) & 86.90 (17.17) & 86.54 (17.39) & 0.802 \\
\hline
\end{tabular}

aThere are 712 ExactCare patients and 4,723 patient-years, and 949,574 comparison patients and 5,091,915 patient-years. For ExactCare patients, baseline is defined as the year before the ExactCare program participation; for non-ExactCare patients, baseline is the second data year. Not all patients were eligible for medication adherence metrics.

${ }^{b}$ Chronic lung disorders include chronic obstructive pulmonary disease, fibrosis of lung, and other chronic lung disorders.

'Renal failure includes stage IV or V chronic kidney disease or end-stage renal disease.

${ }^{d}$ Major mental health conditions include schizophrenia and major depressive, bipolar, and paranoid disorders.

eParalysis includes hemiplegia, quadriplegia, and paraplegia.

'Autoimmune diseases include inflammatory bowel disease, rheumatoid arthritis and inflammatory connective tissue disease, and multiple sclerosis.

$H C C=$ hierarchical condition category; HIV = human immunodeficiency virus; $P D C=$ proportion of days covered; $P M P M=$ per member per month; $S D=$ standard deviation; USD =U.S. dollar.

is in possession of the medication in question. ${ }^{38-42}$

Health Care Utilization. We examined the number of ED visits (excluding those associated with a hospitalization), hospitalizations, and skilled nursing facility (SNF) admissions per 1,000 patient-years, because these are major drivers of health care cost. These events were identified from encounter data using episodes with a place of service in a hospital, SNF, or ED (Supplementary Table 2 for the place of service codes, available in online article), respectively. In the secondary analyses, we also examined hospital and SNF lengths of stay.

Health Care Cost. Total cost of care was computed as per member per month (PMPM) cost for the study population using standardized cost data provided by the data vendor, including both the amount paid by the payer and the amount paid out of pocket by the patient, such as copayments or deductibles. We also separated cost into major cost components: pharmacy cost and medical (nonpharmacy) cost. In the secondary analyses, we further broke down medical costs to outpatient, hospital inpatient, SNF, and other inpatient costs.

\section{ANALYTIC APPROACH}

We used a difference-in-differences analytic approach to estimate program effect on patient outcomes. We first identified the comparison group using propensity score matching to ensure that comparison patients would be as similar as possible to ExactCare patients based on measured characteristics. We then performed a multivariate regression analysis to estimate program effect using multiple years of data for the matched pairs. The comparison group was used to approximate what would have happened had ExactCare patients not participated in the program, condition on that the trends in the outcomes of interest remained similar over time, which is the case upon examination (data not shown).

To perform propensity score matching, we first conducted a logistic regression to model the probability of enrollment in the ExactCare program using data derived before program participation for ExactCare patients and from the baseline year for comparison patients. Predictor variables included patient sociodemographics, individual hierarchical condition category (HCC) medical conditions, health care utilization and cost (see detailed patient characteristics in Table 1), state of residence, and indicators of calendar years. Using the predicted probability of program enrollment, the 2 groups were matched using the nearest 1:2 match approach with a caliper of 0.5 standard deviation of the predicted probability. The baseline data of ExactCare and comparison patients were required to be for the same calendar year. We did not use a 1:3 match because we found that groups were better matched on measured variables after a 1:2 match. Because not all patients 


\section{FIGURE 2 Standardized Differences Between ExactCare and Comparison Patients Before and After Matching}

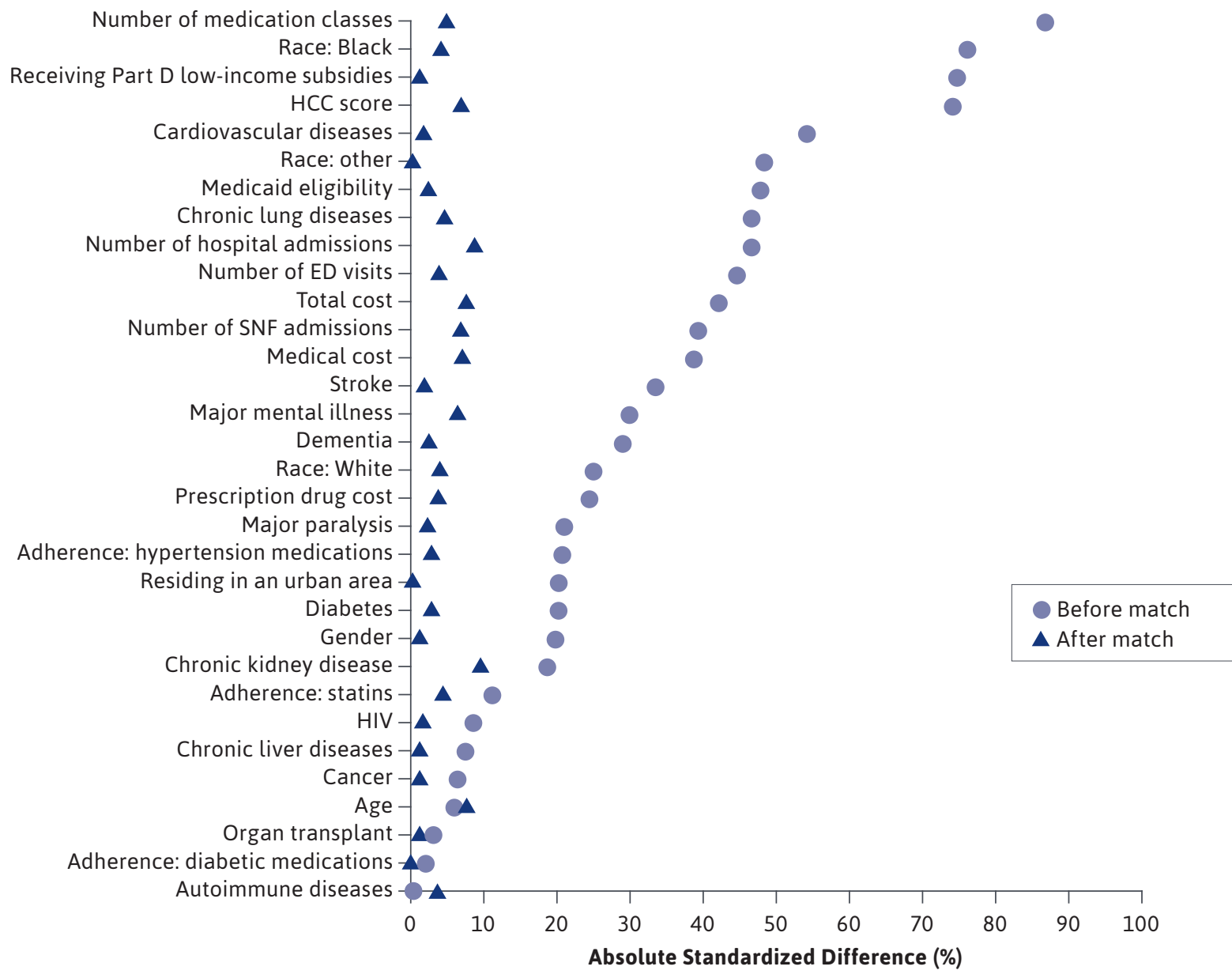

ED=emergency department; HCC = hierarchical condition category; HIV= human immunodeficiency virus; SNF=skilled nursing facility.

were eligible for 3 medication adherence measures, we conducted separate logistic regressions, which included baseline adherence rates, and propensity score matching for each of the 3 measures.

Multivariate models were used to quantify the effect of program participation on outcomes using patient-level fixed effects and Huber-White standard errors, clustered at the patient level. ${ }^{43}$ The key independent variable was the fraction of a year during which a patient was enrolled in the program. Because both patient-level fixed effects and indicators of calendar years are present in the model, the coefficient of the key independent variable represents a difference-in-differences estimate of program effect (see model specifications in the Supplementary Materials, available in online article). In addition, we included additional time-varying characteristics in the model: age, Medicaid enrollment, receipt of Medicare Part D lowincome subsidies, urban residence, state of residence, and HCC community score-a risk adjustor for Medicare Advantage reimbursement purposes.

\section{SECONDARY ANALYSES}

To explore whether the program had a differential effect among subgroups of patients, we conducted secondary analyses among patients who received more medications, were eligible for Medicaid, and had greater comorbidities. Specifically, we conducted propensity score matching and multivariate regressions among patients above the 75th percentile for HCC community score (a score of 3.1 


\section{FIGURE 3 Medication Adherence Rate Changes Associated with the ExactCare Program}

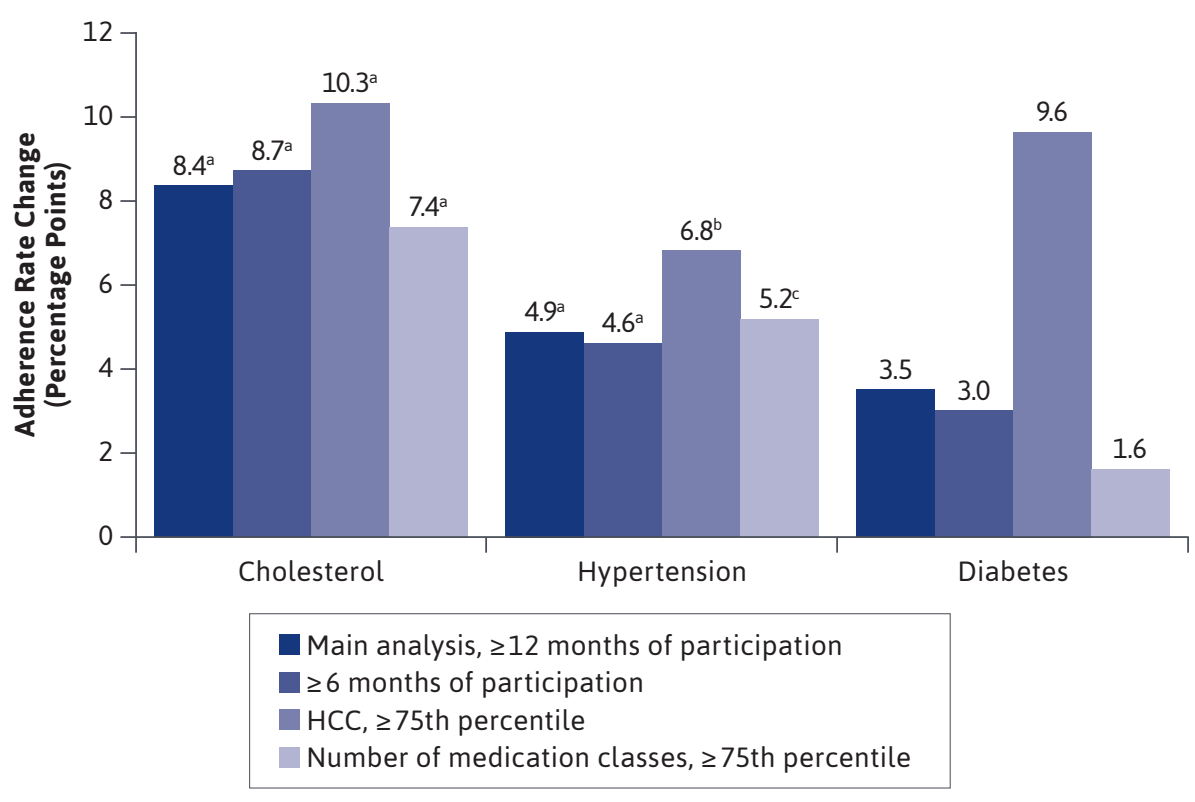

Note: All results are difference-in-differences estimates based on multivariate models with patient-level fixed effects and adjustment for time-varying variables including age, Medicaid eligibility, receipt of Medicare Part D low-income subsidies, HCC scores, and indicators of calendar years and geographic locations (state). The sample sizes of ExactCare patients for cholesterol, hypertension, and diabetes medications, respectively, are as follows: Main analysis, $\geq 12$ months of participation $(n=433,418$, and 108); $\geq 6$ months of participation $(n=673,639$, and 169); HCC, $\geq 75$ th percentile $(n=112,96$, and 20); number of medication classes $\geq 75$ th percentile $(n=138,120$, and 32$)$.

${ }^{a} P<0.001$.

${ }^{\mathrm{b}} \mathrm{P}<0.05$.

${ }^{c} P<0.01$.

$H C C=$ hierarchical condition category.

or greater) of ExactCare patients and among patients above the 75th percentile for the number of medication classes of ExactCare patients (which translated to using 16 or more medication classes in a year). In addition, the main analysis required 12 months or more of ExactCare participation for inclusion in the intervention group, which balanced the need for the intervention to have sufficient duration with excessive limitations on sample size. Given that the optimal duration is unclear, we also conducted analyses that required only 6 months or more of ExactCare participation. To further identify the source of reductions in resource utilization, we also examined between-group differences in hospital length of stay, SNF length of stay, hospital inpatient cost, SNF cost, and other inpatient cost.

\section{Results}

\section{STUDY POPULATION CHARACTERISTICS}

The initial study population included 4,012 ExactCare patients and 2,113,426 comparison patients (Figure 1). After excluding patients younger than aged 18 years and those without
1 or more years of full-year medical and prescription drug coverage, the total number of ExactCare patients decreased to 3,325 , and the number of comparison patients decreased to $1,903,528$. After further limiting the study population to those with baseline data, we identified 712 ExactCare patients and 949,574 potential comparison patients. The ExactCare patients, representing 4,723 memberyears, were followed up for an average of 6.6 years (range: 2-12), including 1 baseline year and 5.6 years after the index year in which the first ExactCare prescription was filled, whereas the potential comparison members, representing 5,091,915 member-years, had an average follow-up of 5.4 years.

As shown in Table 1, before matching, the ExactCare population was similar in age (72.4 vs. 71.8 years; $\mathrm{P}=0.07$ ) and had similar rates of cancer, earlier organ transplant, and autoimmune diseases. However, the ExactCare population was more likely to be female (67.0\% vs. $57.4 \%$ ), Black (41.6\% vs. $10.4 \%)$, and dual eligible for Medicare and Medicaid (25.0\% vs. 7.8\%; $\mathrm{P}<0.001$ for all comparisons). In addition, the ExactCare population was much more likely to have comorbidities as summarized in the substantial difference in the mean HCC score (2.28 vs. $1.27 ; \mathrm{P}<0.001)$ and total cost of care (PMPM total cost of $\$ 3,610$ in ExactCare patients vs. \$1,399 in comparison patients; $\mathrm{P}<0.001$ ).

We matched 701 ExactCare patients to 1,395 comparison members; 11 ExactCare patients were not able to find a matched comparison member, and 7 of the 701 matched ExactCare patients were not able to match with a second comparison member. After matching, the 2 groups are balanced in terms of observable patientlevel characteristics except for the prevalence of renal failure $(5.0 \%$ vs. $3.1 \%$ in ExactCare and comparison patients, respectively; $\mathrm{P}=0.029$; 


\section{FIGURE 4 Cost Changes Associated with the ExactCare Program}

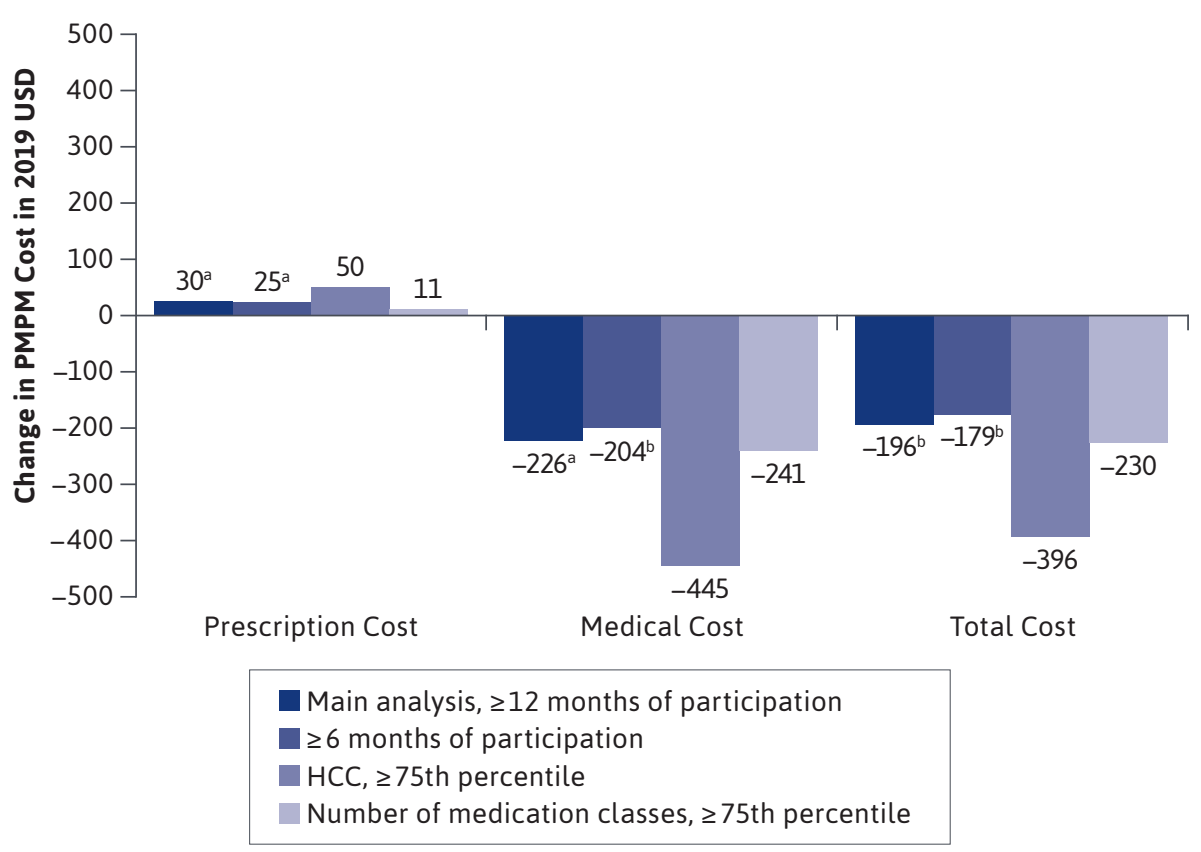

Note: All results are difference-in-differences estimates based on multivariate models with patient-level fixed effects and adjustment for time-varying variables including age, Medicaid eligibility, receipt of Medicare Part D low-income subsidies, HCC scores, and indicators of calendar years and geographic locations (state). The sample sizes of ExactCare patients are as follows: Main analysis, $\geq 12$ months of participation $(n=701) ; \geq 6$ months of participation $(n=1,105)$; HCC, $\geq 75$ th percentile $(n=175)$; number of medication classes, $\geq 75$ th percentile $(n=185)$.

${ }^{a} P<0.01$.

${ }^{\mathrm{b} P}<0.05$.

$H C C=$ hierarchical condition category; USD =U.S. dollar.

Supplementary Table 3 , available in online article). Figure 2 illustrates the absolute standardized difference between ExactCare and comparison patients. After matching, the standardized difference between the 2 groups was greatly reduced for all but 2 variables (age and autoimmune disease) and the absolute difference was $10 \%$ or less for each characteristic.

\section{MEDICATION ADHERENCE}

Figure 3 shows that 1 year of ExactCare participation was associated with statistically significant increases in adherence to statins (8.4 percentage points, $95 \% \mathrm{CI}=6.4$ to 10.4 ) and antihypertensive drugs (4.9 percentage points, $95 \% \mathrm{CI}=2.7$ to 7.1$)$. These results were similar in secondary analyses that varied the definition of ExactCare participation $(\geq 6$ months instead of $\geq 12$ months of program participation) and in analyses restricted to patients with HCC scores $\geq 75$ th percentile and patients with counts of medication classes $\geq 75$ th percentile.

ExactCare was not associated with a statistically significant increase in adherence to diabetes drugs (3.5 percentage points, $95 \% \mathrm{CI}=-0.2$ to 7.2) in the primary and secondary analyses. However, these analyses had the fewest patients (108 patients vs. 433 patients and 418 patients in the primary analyses of adherence for statin and antihypertensive drugs, respectively).

\section{HEALTH CARE UTILIZATION}

One year of ExactCare participation was not associated with statistically significant changes in rates of $\mathrm{ED}$ visits (-21 ED visits per 1,000 memberyears, $95 \% \mathrm{CI}=-207$ to 165 ) or hospital admission rates $(-73$ hospitalizations per 1,000 member-years, $95 \% \mathrm{CI}=-149$ to 3), but it was associated with statistically significant decreases in SNF admission rates $(-67$ SNF stays per 1,000 member-years, $95 \% \mathrm{CI}=-118$ to -16 , Supplementary Figure 1, available in online article). These results were consistent across sensitivity analyses, although the changes in ED visits fluctuated in secondary analyses while never being statistically significant. However, using a similar model for patients with $\geq 12$ months of program participation (Supplementary Table 4, available in online article), we found that 1 year of ExactCare participation was associated with reduced inpatient days (-857 days per 1,000 member-years, $95 \% \mathrm{CI}=-1,588$ to -126$)$ and reduced SNF days $(-1,801$ days per 1,000 member-years, $95 \% \mathrm{CI}=-2,960$ to -643$)$.

\section{HEALTH CARE COSTS}

One year of ExactCare participation was associated with increases in prescription drug costs (\$30 PMPM, 95\% $\mathrm{CI}=\$ 9$ to $\$ 51)$ and decreases in total costs (-\$196 PMPM, 95\% CI $=-\$ 366$ to $-\$ 27)$ and nonprescription costs $(-\$ 226 \quad$ PMPM, $95 \% \quad \mathrm{CI}=-\$ 394 \quad$ to -\$58; Figure 4). These results were consistent across secondary analyses, although analyses restricted to smaller subgroups ( $\mathrm{HCC} \geq 75$ th percentile and number of medication classes $\geq 75$ th percentile) did not show statistically significant differences. In examining specific categories of medical costs (Supplementary Table 4, available in online article), 1 year of 
ExactCare participation was associated with statistically significant decreases in hospital inpatient costs $(-\$ 119$ PMPM, 95\% CI $=-\$ 191$ to $-\$ 47)$ and SNF costs (-\$30 PMPM, $95 \% \mathrm{CI}=-\$ 53$ to $-\$ 8)$, but not outpatient costs $(-\$ 75$ PMPM, 95\% CI $=-\$ 210$ to $\$ 61)$.

\section{Discussion}

Our study is among the first to examine the effect of a comprehensive high-touch medication care management model involving multiple complementary interventions on medication adherence, health care utilization, and cost of care. We demonstrated that receiving 12 or more months of ExactCare medication care management services was associated with patients experiencing statistically significant increases in adherence rates for 2 important and frequently measured classes of medications (antihypertensive drugs and statins) compared with matched nonparticipants.

We also demonstrated that ExactCare participation was associated with statistically significant decreases in total cost of care compared with matched nonparticipants. This reduction corresponds to a 5 percentage point reduction in PMPM costs. ExactCare participation was associated with higher prescription drug costs but lower medical costs, largely attributable to reductions in hospital inpatient cost and SNF cost; this finding is consistent with the aim of the intervention: increasing medication adherence and improving medication management in order to decrease overall costs.

The improvements in total costs seem to be larger than what might be expected solely due to the potential improvements in adherence to the 3 studied drug classes (diabetes drugs, antihypertensive drugs, and statins) and moderate reductions in health care utilization. For example, we found statistically significant reductions in SNF stay rates, but not in ED visit or hospitalization rates, although the ExactCare intervention was associated with lower hospital inpatient days and SNF days.

Three considerations may explain why the reduction in total cost of care remains plausible. First, we were only able to effectively measure adherence in common clinical areas that are subject to existing quality measures and any associated incentives; health plans have already shown improvement on adherence to these 3 medication classes over the past 5 years, ${ }^{44}$ which may limit the ability of any program to improve performance further. However, ExactCare applies similar strategies across all medication classes, which means that there may have also been improvements in adherence to medication classes that we did not measure and for which managed care plans may not be subject to quality measures. Improved adherence in these other (unmeasured) clinical areas could result in reductions in total cost of care and hospitalization costs.

Second, this intervention tested a comprehensive medication management intervention, meaning that it is difficult to say which part of the intervention was most responsible for the benefits we saw. Single interventions such as blister packs, electronic bottle caps, or pharmacist-delivered medication therapy management can improve clinical outcomes ${ }^{7-30}$ but the effect could be enhanced when similar interventions are used in concert. In particular, technologies, such as compliance packaging or electronic reminders and trackers, may have to be used in combination with provider-led interventions such as continual comprehensive medication review in order to generate clinically meaningful effect.

Finally, given the comprehensive nature of the ExactCare program, there might be pathways other than improved medication adherence that could improve patient health outcomes and reduce medication errors, such as monthly medication reviews, regular check-ins with patients, and patient education. Although we were unable to directly observe or measure such pathways to improved outcomes, they do seem likely to have partially accounted for some of the improvements we observed, such as reductions in the number of SNF admissions, hospital and SNF lengths of stay, and hospital inpatient costs and SNF costs.

We noted a trend that the ExactCare program may generate a larger effect in its most clinically complex patients. This is supported by a large, but nonstatistically significant, reduction in total cost of care in patients with an HCC score $\geq 75$ th percentile, which is about twice as large as the reduction observed across all ExactCare patients. It is noteworthy that this apparent enhanced effect of ExactCare was seen among patients with increased illness burden, as measured by HCC score, but not among those taking the most medication classes. The difference suggests that the effect of the ExactCare program may be greater for patients with greater comorbidities rather than solely for those with higher medication counts. Future research is warranted to understand the underlying mechanism, and if such a phenomenon were confirmed, interventions could be directed toward a sicker population determined by measures such as HCC score.

\section{LIMITATIONS}

Our analysis has several limitations. First, the main limitation is that patients who were referred to ExactCare may differ from those not referred in ways that cannot be measured in the data. For example, ExactCare patients may be more likely to live alone, or have subtle signs of undiagnosed dementia, or perhaps poorer control of the same comorbid 
conditions than those patients not given referrals. If this were the case, the inability to measure such variables would tend to diminish the measured effect of the ExactCare intervention, that is, there would be a bias toward the null. On the other hand, providers might avoid referring the sickest or least compliant patients to ExactCare if they felt that the patients would not benefit from the intervention, which would inflate the measured effect. We mitigated this risk by using propensity score methods to match intervention patients to comparison patients on a series of baseline characteristics including baseline costs, but the 2 groups may still not be balanced in terms of the risk of incurring health care utilization and medical spending.

Second, we had a relatively limited sample of 701 intervention patients, which reduced our ability to detect some differences, and to preserve the sample size, we used the fraction of a year with program participation as the dependent variable and assumed the effect to be linear in time.

Third, the data came from 1 insurer, and thus the generalizability of the study results may be limited if standard disease management practices (i.e., practices used among the comparison group) differ from those of the community. Including patients from multiple payers in future analyses could improve generalizability.

Fourth, the study population included a high percentage of dropouts, and we could not ascertain the reason for their leaving ExactCare. If the reasons were correlated with worse care (i.e., death or transition to different insurers due to dissatisfaction with their insurer), our estimates would likely be biased toward greater savings. As a result, our findings may not generalize outside of the population studied in this analysis (i.e., longer-term ExactCare patients).
Finally, the comparison group likely included patients who participated in other medication adherence or disease management programs, which would also bias toward the null. In fact, as our data were drawn from one of the largest private insurers in the United States, the effectiveness of its management of Medicare Advantage patients may be above the average. If so, this would have caused the analysis to underestimate the effect of ExactCare in comparison to having no adherence support or disease management programs at all.

\section{Conclusions}

Our analysis shows that ExactCare's medication care management model was associated with total cost of care reductions of approximately $\$ 2,400$ per member per year in relation to matched comparison patients and better adherence to 2 drug classes. Further research is warranted to confirm this finding.

\section{DISCLOSURES}

This study was funded by ExactCare Pharmacy under a contract with RAND that grants the study authors sole responsibility for data management, study design, data analysis, manuscript drafting, and the decision to publish. The sponsor had no role in the study design and manuscript drafting. All data analysis was conducted by the study authors. A draft manuscript was reviewed by the sponsor, but the study authors made final decisions regarding the content and study conclusions. Shetty, Chen, and Liu are employed by RAND. Rose has nothing to disclose.

\section{REFERENCES}

1. Kantor ED, Rehm CD, Haas JS, Chan AT, Giovannucci EL. Trends in prescription drug use among adults in the United States from 1999-2012. JAMA. 2015;314(17):1818-31.
2. Charlesworth CJ, Smit E, Lee DS, Alramadhan F, Odden MC. Polypharmacy among adults aged 65 years and older in the United States: 1988-2010. J Gerontol A Biol Sci Med Sci. 2015;70(8):989-95.

3. Diez-Manglano J, Gimenez-Lopez M, Garces-Horna V, et al. Excessive polypharmacy and survival in polypathological patients. Eur J Clin Pharmacol. 2015;71(6):733-39.

4. Gnjidic D, Hilmer SN, Blyth FM, et al. High-risk prescribing and incidence of frailty among older communitydwelling men. Clin Pharmacol Ther. 2012;91(3):521-28.

5. O'Dwyer M, Peklar J, McCallion P, McCarron M, Henman MC. Factors associated with polypharmacy and excessive polypharmacy in older people with intellectual disability differ from the general population: a cross-sectional observational nationwide study. BMJ Open. 2016;6(4):e010505.

6. Conn VS, Ruppar TM, Chan KC, Dunbar-Jacob J, Pepper GA, De Geest S. Packaging interventions to increase medication adherence: systematic review and meta-analysis. Curr Med Res Opin. 2015;31(1):145-60.

7. Rootes-Murdy K, Glazer KL, Van Wert MJ, Mondimore FM, Zandi PP. Mobile technology for medication adherence in people with mood disorders: A systematic review. J Affect Disord. 2018;227:613-17.

8. Rosen MI, Rigsby MO, Salahi JT, Ryan CE, Cramer JA. Electronic monitoring and counseling to improve medication adherence. Behav Res Ther. 2004;42(4):409-22.

9. Dayer L, Heldenbrand S, Anderson P, Gubbins PO, Martin BC. Smartphone medication adherence apps: potential benefits to patients and providers. J Am Pharm Assoc (2003). 2013;53(2):172-81. 
10. Farmer AJ, McSharry J, Rowbotham S, McGowan L, Ricci-Cabello I, French DP. Effects of interventions promoting monitoring of medication use and brief messaging on medication adherence for people with Type 2 diabetes: a systematic review of randomized trials. Diabet Med. 2016;33(5):565-79.

11. Mehta SJ, Volpp KG, Troxel AB, et al. Electronic pill bottles or bidirectional text messaging to improve hypertension medication adherence (way 2 text): a randomized clinical trial. J Gen Intern Med. 2019;34(11):2397-404.

12. Moore DJ, Poquette A, Casaletto KB, et al. Individualized texting for adherence building (iTAB): improving antiretroviral dose timing among HIV-infected persons with co-occurring bipolar disorder. AIDS Behav. 2015;19(3):459-71.

13. Nglazi MD, Bekker LG, Wood R, Hussey GD, Wiysonge CS. Mobile phone text messaging for promoting adherence to anti-tuberculosis treatment: a systematic review. BMC Infect Dis. 2013;13:566.

14. Vervloet M, Linn AJ, van Weert JC, de Bakker DH, Bouvy ML, van Dijk L. The effectiveness of interventions using electronic reminders to improve adherence to chronic medication: a systematic review of the literature. J Am Med Inform Assoc. 2012;19(5):696-704.

15. Devine S, Vlahiotis A, Sundar H. A comparison of diabetes medication adherence and healthcare costs in patients using mail order pharmacy and retail pharmacy. J Med Econ. 2010;13(2):203-11.

16. Duru OK, Schmittdiel JA, Dyer WT, et al. Mail-order pharmacy use and adherence to diabetes-related medications. Am J Manag Care. 2010;16(1):33-40.

17. Tiktin M, Celik S, Berard L.

Understanding adherence to medications in type 2 diabetes care and clinical trials to overcome barriers: a narrative review. Curr Med Res Opin. 2016;32(2):277-87.

18. Cobb CD. Optimizing medication use with a pharmacist-provided comprehensive medication management service for patients with psychiatric disorders. Pharmacotherapy. 2014;34(12):1336-40.
19. Moore JM, Shartle D, Faudskar L, Matlin OS, Brennan TA. Impact of a patient-centered pharmacy program and intervention in a high-risk group. J Manag Care Pharm. 2013;19(3):228-36. doi: 10.18553/jmcp.2013.19.3.228

20. Dokbua S, Dilokthornsakul P, Chaiyakunapruk N, Saini B, Krass I, Dhippayom T. Effects of an asthma selfmanagement support service provided by community pharmacists: a systematic review and meta-analysis. J Manag Care Spec Pharm. 2018;24(11):1184-96. doi: 10.18553/jmcp.2018.24.11.1184

21. Hatah E, Braund R, Tordoff J, Duffull SB. A systematic review and meta-analysis of pharmacist-led fee-for-services medication review. Br J Clin Pharmacol. 2014;77(1):102-15.

22. Jokanovic N, Tan EC, Sudhakaran S, et al. Pharmacist-led medication review in community settings: an overview of systematic reviews. Res Social Adm Pharm. 2017;13(4):661-85.

23. Milosavljevic A, Aspden T, Harrison J. Community pharmacist-led interventions and their impact on patients' medication adherence and other health outcomes: a systematic review. Int J Pharm Pract. 2018;26(5):387-97.

24. Mubarak N, Hatah E, Khan TM, Zin CS. A systematic review and meta-analysis of the impact of collaborative practice between community pharmacist and general practitioner on asthma management. J Asthma Allergy. 2019;12:109-53.

25. Murphy JA, Schroeder MN, Rarus RE, Yakubu I, McKee SOP, Martin SJ.

Implementation of a cardiac transitions of care pilot program: a prospective study of inpatient and outpatient clinical pharmacy services for patients with heart failure exacerbation or acute myocardial infarction. J Pharm Pract. 2019;32(1):68-76.

26. Persell SD, Karmali KN, Lazar D, et al. Effect of electronic health record-based medication support and nurse-led medication therapy management on hypertension and medication selfmanagement: a randomized clinical trial. JAMA Intern Med. 2018;178(8):1069-77.
27. Rotta I, Salgado TM, Silva ML, Correr CJ, Fernandez-Llimos F. Effectiveness of clinical pharmacy services: an overview of systematic reviews (2000-2010). Int J Clin Pharm. 2015;37(5):687-97.

28. Yuan C, Ding Y, Zhou K, Huang Y, Xi X. Clinical outcomes of community pharmacy services: A systematic review and meta-analysis. Health Soc Care Community. 2019;27(5):e567-87.

29. Zhu J, Ayer G, Kirkham HS, et al. Outcome evaluation of a subcutaneous immunoglobulin clinical management program. J Res Pharm Pract. 2019;8(2):52-63.

30. Zhu J, Kirkham HS, Ayer G, et al. Clinical and economic outcomes of a "high-touch" clinical management program for intravenous immunoglobulin therapy. Clinicoecon Outcomes Res. 2018;10:1-12.

31. Choudhry NK, Krumme AA, Ercole PM, et al. Effect of reminder devices on medication adherence: the REMIND randomized clinical trial. JAMA Intern Med. 2017;177(5):624-31.

32. Volpp KG, Troxel AB, Mehta SJ, et al. Effect of electronic reminders, financial incentives, and social support on outcomes after myocardial infarction: the HeartStrong randomized clinical trial. JAMA Intern Med. 2017;177(8):1093-01.

33. Fortney JC, Pyne JM, Edlund MJ, et al. A randomized trial of telemedicine-based collaborative care for depression. J Gen Intern Med. 2007;22(8):1086-93.

34. Gordon JS, Armin JS, Cunningham JK, Muramoto ML, Christiansen SM, Jacobs TA. Lessons learned in the development and evaluation of RxCoach, an mHealth app to increase tobacco cessation medication adherence. Patient Educ Couns. 2017;100(4):720-27.

35. Steinkamp JM, Goldblatt N, Borodovsky JT, et al. Technological interventions for medication adherence in adult mental health and substance use disorders: a systematic review. JMIR Ment Health. 2019;6(3):e12493. 
36. ExactCare. Medication management for complex, high-risk members. 2019. Accessed January 13, 2021. https://www. exactcarepharmacy.com/payers/

37. Chang A, Gillespie C. Paper 3560-2015: A SAS macro to calculate the PDC adjustment of inpatient stays. 2015. Accessed January 13, 2021. https://support.sas. com/resources/papers/proceedings15/3560-2015.pdf

38. PQA Alliance. Measures sets. 2018. Accessed January 13, 2021. https://www. pqaalliance.org/measures-overview

39. PQA Alliance. PQA quality measures used in Centers for Medicare and Medicaid Services (CMS) Programs. 2020. Accessed January 13, 2021. https://www. pqaalliance.org/assets/Measures/PQA Measures in CMS Programs.pdf
40. Steiner JF, Koepsell TD, Fihn SD, Inui TS. A general method of compliance assessment using centralized pharmacy records: description and validation. Med Care. 1988;26(8):814-23.

41. Ho PM, Bryson CL, Rumsfeld JS. Medication adherence: its importance in cardiovascular outcomes. Circulation. 2009;119(23):3028-35.

42. Bryson CL, Au DH, Young B, McDonell $\mathrm{MB}$, Fihn SD. A refill adherence algorithm for multiple short intervals to estimate refill compliance (ReComp). Med Care. 2007;45(6):497-504.

43. Huber PJ. Robust estimation of a location parameter. Ann Math Stat. 1964:73-101.
44. Centers for Medicare \& Medicaid Services. 2018 national impact assessment of the Centers for Medicare \& Medicaid Services (CMS) quality measures report. February 11, 2020. Accessed January 13, 2021. https://www.cms.gov/Medicare/ Quality-Initiatives-Patient-AssessmentInstruments/QualityMeasures/ National-Impact-Assessment-of-theCenters-for-Medicare-and-MedicaidServices-CMS-Quality-Measures-Reports 